\title{
Prävention der Herzinsuffizienz
}

G. Noll*

\section{Zusammenfassung}

Die Herzinsuffizienz hat in den letzten Jahrzehnten deutlich zugenommen. Trotz neuer effizienter Behandlungen sind Mortalität und Morbidität herzinsuffizienter Patienten nach wie vor hoch, die Lebensqualität stark eingeschränkt. Daten der letzten Jahre zeigen, dass eine asymptomatische linksventrikuläre Dysfunktion bei 1 bis 12,5\% der Erwachsenen vorkommt, wobei Alter und die bekannten kardiovaskulären Risikofaktoren eine wichtige Rolle spielen. Obschon ein Routinescreening zur Erfassung der linksventrikulären Dysfunktion sich aufgrund ökonomischer Aspekte nicht rechtfertigen lässt, ist aber die strikte Vermeidung bzw. konsequente Behandlung der bekannten Risikofaktoren für eine Herzinsuffizienz dringlich, wobei bei Patienten mit niedrigem Risiko nichtmedikamentöse Massnahmen wie Gewichtsreduktion, Nikotinstopp, sportliche Aktivität und eine mediterrane Diät im Vordergrund stehen. Bei Hochrisikopatienten und beim Vorliegen einer eingeschränkten linksventrikulären Funktion ist eine medikamentöse Therapie, vorzugsweise mit einem ACE-Hemmer, indiziert.

\footnotetext{
* Dieser Artikel erscheint im Rahmen des Aufklärungsprogramms «Herzinsuffizienz» der Schweizerischen Herzstiftung. Er wird im Namen der Projektgruppe «Herzinsuffizienz» der Schweizerischen Herzstiftung und der Arbeitsgruppe «Herzinsuffizienz» der Schweizerischen Gesellschaft für Kardiologie publiziert.

Die folgenden Mitglieder haben zu dieser Publikation beigetragen: P. Buser, Basel; H.-P. Brunner, Basel; D. Conen, Aarau (Vertreter der SGIM); R. Darioli, Lausanne; O. M. Hess, Bern; P. Mohacsi, Bern; R. Mordasini, Bern; G. Noll, Zürich (Verfasser); P. Périat, Riehen (Vertreter der SGAM), M. Pfisterer, Basel; R. Lerch, Genf, T. Szucs,

Basel; P. Trigo-Trindade, Genf.
}

Korrespondenz:

Prof. Dr. Georg Noll

Mercator-Professor

HerzKreislaufZentrum

Kardiologie

UniversitätsSpital

CH-8091 Zürich

\section{Epidemiologie und Risikofaktoren}

Trotz Abnahme der Häufigkeit der koronaren Herzkrankheit hat die Prävalenz der Herzinsuffizienz in den letzten Jahrzehnten zugenommen und beträgt momentan etwa $1-2 \%$, bei über 65jährigen 6-10\% [1]. Dies wird vor allem auf die bessere Behandlung der Myokardinfarkte und die veränderte Altersstruktur der Bevölkerung zurückgeführt. Eine kürzliche Analyse hat ergeben, dass das Risiko, an einer Herzinsuffizienz zu erkranken, für alle Altersgruppen ca. $20 \%$ beträgt [2]. Damit stellt die Herzinsuffizienz ein enormes gesundheitspolitisches und ökonomisches Problem dar, sind Patienten mit Herzinsuffizienz doch häufig hospitalisiert, nehmen auch andere Leistungen in Anspruch und verursachen deshalb enorme Kosten. In grossen epidemiologischen Studien konnten verschiedene Risikofaktoren für die Entwicklung einer Herzinsuffizienz identifiziert werden (Tab. 1). Obschon die Herzinsuffizienz ein heterogenes Krankheitsbild mit verschiedenen Ätiologien darstellt, kann die Mehrzahl der Fälle auf drei wichtige zugrundeliegende Faktoren zurückgeführt werden: 1. die koronare Herzkrankheit; 2. die arterielle Hypertonie (und sekundär auch die linksventrikuläre Hypertrophie) und 3. den Diabetes mellitus. In der Framingham-Studie konnte gezeigt werden, dass koronare Herzkrankheit und arterielle Hypertonie für drei Viertel der Herzinsuffizienzfälle verantwortlich sind [3]. Patienten mit einem Blutdruck von systolisch $>160 \mathrm{~mm} \mathrm{Hg}$ oder diastolisch $>90 \mathrm{~mm} \mathrm{Hg}$ weisen ein $2 \mathrm{fach}$ höheres Risiko auf, an einer Herzinsuffizienz zu erkranken, als Patienten mit einem Blutdruck von $<140 / 90$ mm Hg [2, 4]. Das Vorliegen einer linksventrikulären Hypertrophie steigert das Risiko zusätzlich. Dies ist von enormer Bedeutung, weist die arterielle Hypertonie doch eine sehr hohe Prävalenz in der Bevölkerung auf. Epidemiologische Studien haben gezeigt, dass Patienten mit einem Diabetes mellitus ein besonders hohes Risiko kardiovaskulärer Komplikationen aufweisen, welches demjenigen von Nicht-Diabetikern mit durchgemachtem Herzinfarkt vergleichbar ist [5]. Bei ungefähr 40\% der Patienten mit Herzinsuffizienz liegt entweder ein Diabetes mellitus oder eine eingeschränkte Glukosetoleranz vor [6-8]. Bei Patienten mit eingeschränkter linksventrikuärer Funktion (Auswurffraktion $<35 \%$ ) war das Vorliegen eines Diabetes mellitus ein unabhängiger Prädiktor für Mortalität und die Entwicklung einer Herzinsuffizienz [9]. Weitere unabhängige Risikofaktoren sind Rauchen, Übergewicht und das Vorliegen einer Albu-

Tabelle 1

Risikofaktoren für die Entwicklung einer Herzinsuffizienz.

Eingeschränkte linksventrikuläre Funktion; koronare Herzkrankheit; arterielle Hypertonie, linksventrikuläre Hypertrophie; Diabetes mellitus;

Adipositas;

Dyslipidämie;

Rauchen;

körperliche Inaktivität;

Mikroalbuminurie;

Familienanamnese für Kardiomyopathie;

Niereninsuffizienz. 
Tabelle 2

Stadieneinteilung der Herzinsuffizienz gemäss der American Heart Association.

\begin{tabular}{|c|c|c|}
\hline Stadium & Beschreibung & Beispiel \\
\hline A & $\begin{array}{l}\text { Patienten mit hohem Risiko für eine Herzinsuffizienz ohne strukturelle } \\
\text { Veränderungen von Perikard, Myokard und Klappen, ohne Zeichen } \\
\text { oder Symptome einer Herzinsuffizienz }\end{array}$ & $\begin{array}{l}\text { Arterielle Hypertonie, koronare Herzkrankheit, Diabetes mellitus. } \\
\text { Alkoholabusus oder kardiotoxische Medikamente in der Anamnese. } \\
\text { Durchgemachtes rheumatisches Fieber. Familienanamnese für } \\
\text { Kardiomyopathie }\end{array}$ \\
\hline B & $\begin{array}{l}\text { Patienten mit struktureller Herzkrankheit, die mit einer Herzinsuffizienz } \\
\text { assoziiert ist, welche aber nie Zeichen oder Symptome einer } \\
\text { Herzinsuffizienz gezeigt haben }\end{array}$ & $\begin{array}{l}\text { Linksventrikuläre Hypertrophie oder Fibrose; linksventrikuläre } \\
\text { Dilatation oder Hypokinesie; asymptomatische valvuläre Herzkrank- } \\
\text { heit; durchgemachter Myokardinfarkt }\end{array}$ \\
\hline C & $\begin{array}{l}\text { Patienten mit struktureller Herzkrankheit, die momentan oder früher } \\
\text { Symptome einer Herzinsuffizienz aufweisen/aufgewiesen haben }\end{array}$ & $\begin{array}{l}\text { Dyspnoe oder Leistungseinschränkung infolge linksventrikulärer } \\
\text { Dysfunktion; Patienten, die unter Therapie für eine Herzinsuffizienz } \\
\text { asymptomatisch sind }\end{array}$ \\
\hline D & $\begin{array}{l}\text { Patienten mit fortgeschrittener struktureller Herzkrankheit, die trotz } \\
\text { maximaler medikamentöser Behandlung in Ruhe symptomatisch sind } \\
\text { und spezialisierter Interventionen bedürfen }\end{array}$ & $\begin{array}{l}\text { Patienten, die häufig wegen Herzinsuffizienz hospitalisiert sind; } \\
\text { Patienten, die hospitalisiert auf eine Transplantation warten oder eine } \\
\text { dauernde intravenöse medikamentöse Behandlung benötigen. } \\
\text { Patienten, die eine mechanische Unterstützung brauchen }\end{array}$ \\
\hline
\end{tabular}

minurie [10-12]. Ist eine asymptomatische Einschränkung der linksventrikulären Funktion vorhanden, ist das Risiko einer Herzinsuffizienz ebenfalls stark erhöht, wobei die Ätiologie der linksventrikulären Dysfunktion eine wichtige Rolle spielt. Bei Vorliegen einer koronaren Herzkrankheit ist das Risiko deutlich höher. Die American Heart Association (AHA) und das American College of Cardiology (ACC) haben im Jahr 2001 eine neue Stadieneinteilung der Herzinsuffizienz eingeführt, die nicht nur den Schweregrad der Herzinsuffizienz berücksichtigt, sondern auch Patienten mit erhöhtem Risiko einschliesst (Tab. 2) [1]. Die präklinischen Stadien A und B zeigen das Potential für präventive Interventionen auf.

\section{Pathophysiologische Veränderungen, welche zur Herzinsuffizienz führen}

Sowohl bei Patienten mit asymptomatischer wie auch symptomatischer linksventrikulärer Funktionsstörung kommt es zu einer neurohumoralen Aktivierung, welche durch Überlastung der Ventrikel und durch direkte Wirkung der freigesetzten Hormone zu einer progressiven Myokardschädigung führt. Der dadurch bedingte Umbau des linksventrikulären Myokardes, welcher mit Hypertrophie, Kammerdilatation und weiterer Reduktion der kontraktilen Funktion einhergeht, wird als «Remodeling» bezeichnet. Das Ausmass der systolischen Dysfunktion korreliert mit Plasmawerten von Neurohormonen. So ist bekannt, dass Noradrenalin im Plasma auch im asymptomatischen Stadium bei Patienten mit linksventrikulärer Dysfunktion (Stadium B der Einteilung nach AHA/ACC) ein unabhän- giger prädiktiver Faktor für Mortalität und Herzinsuffizienz ist [13]. Nach einem Myokardinfarkt beginnt der Prozess des Remodelings früh nach dem akuten Ereignis. Viele Patienten sind aber trotz Vergrösserung des Ventrikels über Jahre asymptomatisch, haben jedoch ein deutlich erhöhtes Risiko, in Zukunft eine Herzinsuffizienz zu entwickeln. Für diesen Prozess des strukturellen Umbaus des Myokards, dessen genaue Mechanismen noch unklar sind, spielt ein erhöhter Wandstress sowie eine Aktivierung des ReninAngiotensin-Aldosteron-Systems eine Rolle.

\section{Prävention des Auftretens einer Herzinsuffizienz}

Verschiedene grosse klinische Studien haben gezeigt, dass Angiotensin-Converting-EnzymInhibitoren (ACE-Hemmer), $\beta$-Blocker und, im fortgeschrittenen Stadium, Aldosteron-Antagonisten zu einer Reduktion der Mortalität bei Patienten mit symptomatischer Herzinsuffizienz führen. Dennoch bleibt die Prognose bei diesen Patienten schlecht, weshalb präventiven Strategien zur Verhinderung der Herzinsuffizienz unbedingt mehr Beachtung geschenkt werden muss. Abhängig vom Stadium im meist langsam fortschreitenden Krankheitsprozess, welcher zur manifesten Herzinsuffizienz führt (Tab. 2), müssen unterschiedliche Strategien angewendet werden (Abb. 1). Da die koronare Herzkrankheit den wichtigsten Risikofaktor für die Herzinsuffizienz darstellt, steht bei gesunden Personen (Stadium A der Einteilung nach AHA/ACC) die Prävention der Arteriosklerose mittels nichtmedikamentöser Massnahmen wie Nikotinabstinenz, mediterraner Diät, körperlicher Aktivität 
und Gewichtsreduktion im Vordergrund. Bei erhöhtem Risiko (nach Herzinfarkt, mit oder ohne Einschränkung der linksventrikulären Funktion, arterieller Hypertonie) ist zusätzlich eine medikamentöse Behandlung indiziert [1, 14].

Die Prävalenz der asymptomatischen Einschränkung der linksventrikulären Funktion (Stadium B der Einteilung nach AHA/ACC) beträgt $0,9-12,9 \%$, wobei sie bei Patienten mit koronarer Herzkrankheit deutlich höher liegt [15]. Aufgrund der Daten randomisierter Studien muss davon ausgegangen werden, dass für diese Patienten das Risiko, eine Herzinsuffizienz zu entwickeln, 5-20\% pro Jahr beträgt [16-20]. Deshalb wäre eine frühe Erfassung der Funktionseinschränkung des linken Ventrikels wünschenswert. Aus Kostengründen kommt eine Echokardiographie als Screeningmethode nicht in Frage. Klinische Studien werden zeigen, ob sich die Bestimmung des Brain-Natriuretic-Peptide, das bei Dehnung der Kammerwand aus dem Myokard freigesetzt wird, für die Früherfassung der linksventrikulären Funktionseinschränkung eignet.

\section{Medikamentöse Interventionen bei verschiedenen Patientenpopulationen}

\section{Behandlung der Hypertonie}

Die Behandlung der arteriellen Hypertonie reduziert das Risiko für eine Herzinsuffizienz deutlich (Tab. 3). Mehrere placebokontrollierte Studien dokumentieren eine Risikoreduktion von 29 bis 51\% [21-28]. Vergleichende Untersuchungen weisen darauf hin, dass insbesondere der Einsatz von ACE-Hemmern / AngiotensinRezeptor-Antagonisten oder Diuretika einer Behandlung mit einem Kalzium-Antagonisten überlegen zu sein scheint. In der kürzlich publizierten ALLHAT-Studie, die die Behandlung einer arteriellen Hypertonie mit verschiedenen Medikamenten verglich (Chlorthalidon, Lisinopril, Doxazosin, Amlodipin), konnte gezeigt werden, dass der primäre Endpunkt (nicht-tödlicher oder tödlicher Herzinfarkt oder Hirnschlag) in den untersuchten Gruppen nicht unterschiedlich war [27]. Im Vergleich zur Behandlung mit Chlorthalidon waren jedoch während der Initialphase der Behandlung in den mit ACE-

Abbildung 1

Prävention und Therapie der Herzinsuffizienz (HI) entsprechend der Stadieneinteilung gemäss der American Heart Association.

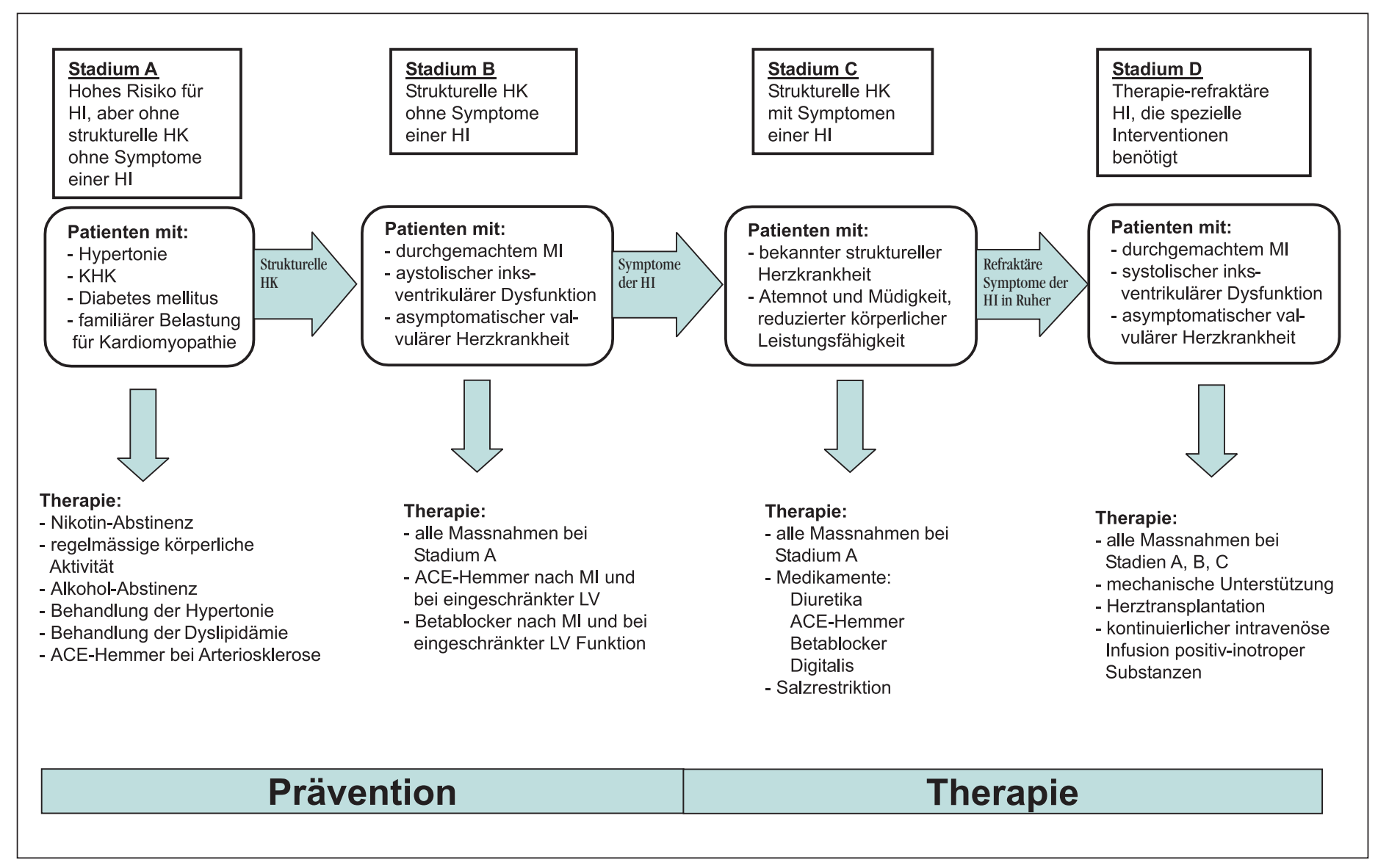


Tabelle 3

Medikamentöse Behandlung zur Reduktion des Herzinsuffizienzrisikos.

\begin{tabular}{|c|c|c|c|c|c|}
\hline Studie & Therapie & $\mathbf{n}$ & $\begin{array}{r}\text { FU } \\
\text { (Jahre) }\end{array}$ & $\begin{array}{r}\text { Risiko } \\
(\%)\end{array}$ & $\begin{array}{l}\text { Änderung des relativen } \\
\text { Risikos (\%) }\end{array}$ \\
\hline \multicolumn{6}{|l|}{ Arterielle Hypertonie } \\
\hline \multirow{2}{*}{ STOP [21] } & Betablocker (+HTCZ) & 812 & 2,1 & 2,3 & -51 (*vs Placebo) \\
\hline & Placebo & 815 & & 4,8 & \\
\hline \multirow[t]{2}{*}{ SHEP [23] } & Chlorthalidon (+Atenolol) & 2365 & 4,5 & 2,3 & -51 (*vs Placebo) \\
\hline & Placebo & 2371 & & 4,4 & \\
\hline \multirow{2}{*}{ Syst-Eur [24] } & Nitrendipine & 2398 & 2 & 1,2 & $-36(n s)$ \\
\hline & Placebo & 2297 & & 1,8 & \\
\hline \multirow[t]{3}{*}{ STOP-2 [22] } & Betablocker/HTCZ & 2213 & 4 & 8,0 & \\
\hline & ACE-Hemmer & 2205 & & 6,8 & -22 (*vs Kalzium- \\
\hline & Kalzium-Antagonisten & 2196 & & 8,2 & \\
\hline \multirow[t]{2}{*}{ INSIGHT [25] } & Nifedipine GITS & 3157 & 3 & 0,8 & \\
\hline & Co-Amilorid & 3164 & & 0,4 & $\begin{array}{l}-50 \text { (*vs Kalzium- } \\
\text { Antagonist) }\end{array}$ \\
\hline \multirow[t]{2}{*}{ NORDIL [40] } & Diltiazem & 5410 & 4,5 & 1,1 & \\
\hline & Diuretikum/Betablocker & 5471 & & 1,0 & (ns) \\
\hline \multirow[t]{2}{*}{ CAPP [41] } & Captopril & 5492 & 6,1 & 1,4 & \\
\hline & Betablocker /Diuretikum & 5493 & & 1,2 & (ns) \\
\hline \multirow{2}{*}{$\begin{array}{l}\text { ALLHAT [26] } \\
\text { (vorzeitig abgebrochen) }\end{array}$} & Chlorthalidon & 15268 & 3,3 & 4,5 & \\
\hline & Doxazosin & 9067 & & 8,1 & +80 (*vs Chlorthalidon) \\
\hline \multirow[t]{3}{*}{ ALLHAT [27] } & Chlorthalidon & 15255 & 4,6 & 5,7 & \\
\hline & Lisinopril & 9054 & & 6,8 & +19 (*vs Chlorthalidon) \\
\hline & Amlodipine & 9048 & & 7,8 & +38 (*vs Chlorthalidon) \\
\hline \multirow[t]{2}{*}{ ANPB [42] } & Diuretikum & 3039 & 4,1 & 2,5 & \\
\hline & ACE-Hemmer & 3044 & & 2,2 & -15 (ns) \\
\hline \multirow{2}{*}{ LIFE [28] } & Losartan & 4605 & 4,8 & 3,3 & -3 (ns) \\
\hline & Atenolol & 4588 & & 3,5 & \\
\hline \multicolumn{6}{|l|}{ Diabetes } \\
\hline \multirow{2}{*}{ UKPDS [32] } & $\mathrm{BD}<150 / 85$ & 758 & 8,4 & 2,8 & $-56(n s)$ \\
\hline & $\mathrm{BD}<180 / 105$ & 390 & & 6,2 & \\
\hline \multirow[t]{2}{*}{ LIFE (Hypertonie) [43] } & Losartan & 586 & 4,8 & 5,5 & -41 (*vs Atenolol) \\
\hline & Atenolol & 609 & & 9,0 & \\
\hline \multirow{2}{*}{$\begin{array}{l}\text { RENAAL (Nephropathie) } \\
\text { [33] }\end{array}$} & Losartan & 751 & 3,4 & 11,9 & $-32\left({ }^{*} v s\right.$ Placebo $)$ \\
\hline & Placebo & 762 & & 16,7 & \\
\hline \multicolumn{6}{|l|}{ Patienten mit Arteriosklerose } \\
\hline \multirow[t]{2}{*}{ HOPE [34] } & Ramipril & 4645 & 4,5 & 9,0 & $-23\left({ }^{*}\right.$ vs Placebo $)$ \\
\hline & Placebo & 4652 & & 11,5 & \\
\hline \multicolumn{6}{|c|}{ Eingeschränkte LV-Funktion } \\
\hline \multirow[t]{2}{*}{ SOLVD [16] } & Enalapril & 2111 & 3,1 & 20,7 & -37 (*vs Placebo) \\
\hline & Placebo & 2117 & & 30,2 & \\
\hline \multicolumn{6}{|l|}{ Akutes Koronarsyndrom } \\
\hline \multirow[t]{2}{*}{ CURE [36] } & Clopidogrel & 6259 & 0,75 & 3,7 & -18 (*vs Placebo) \\
\hline & Placebo & 6303 & & 4,4 & \\
\hline \multicolumn{6}{|l|}{ Hypercholesterinämie } \\
\hline \multirow[t]{2}{*}{$4-S[31]$} & Simvastatin & 2221 & 5,4 & 8,3 & -21 ( ${ }^{*} v s$ Placebo $)$ \\
\hline & Placebo & 222 & & & \\
\hline
\end{tabular}


Hemmern, Kalzium-Antagonisten oder Alphablockern behandelten Gruppen die Frequenz von Herzinsuffizienz offenbar signifikant höher als in der mit Chlorthalidon behandelten Gruppe. Dieses Phänomen ist unklar und liegt möglicherweise im Design der Studie, ist doch eine Mehrzahl der Patienten nicht therapie-naiv, sondern vorgängig mit einem Diuretikum behandelt worden [29]. Auch könnte es damit zu tun haben, dass der Diagnose der Herzinsuffizienz keine strengen Kriterien zugrundelagen und eine konsequente Kontrolle der berichteten Fälle unterblieb. Metaanalysen lassen vermuten, dass Patienten, die mit Kalzium-Antagonisten behandelt werden, im Vergleich zu anderen Antihypertensiva geringgradig häufiger eine Herzinsuffizienz entwickeln [30]. Auch wenn diskrete Unterschiede zwischen verschiedenen Antihypertensiva hinsichtlich des Auftretens einer Herzinsuffizienz vorhanden sind, muss doch darauf hingewiesen werden, dass primär durch die Blutdrucksenkung die Prognose der Patienten verbessert wird, wofür fast immer bestimmte Kombinationen von Medikamenten, die in ALLHAT nicht angewendet werden konnten, benötigt werden.

\section{Dyslipidämie}

In der 4-S-Studie bei Patienten mit koronarer Herzkrankheit und Hypercholesterinämie wurde das Auftreten einer Herzinsuffizienz durch den Einsatz von Simvastatin von 10,3 auf $8,3 \%$ um $21 \%$ reduziert [31]. Weitere Studien sind nötig, um die Mechanismen dieser Risikoreduktion aufzuklären.

\section{Diabetes mellitus}

Bei Patienten mit Diabetes mellitus ist neben der Blutzuckereinstellung vor allem eine effiziente Blutdrucksenkung für die Prävention makrovaskulärer Ereignisse entscheidend. Die UKPDS (United Kingdom Prospective Diabetes Study) hat gezeigt, dass eine bessere Blutdruckkontrolle nicht nur die Anzahl Myokardinfarkte, sondern auch das Risiko einer Herzinsuffizienz reduziert [32].

Bei Diabetikern mit Nephropathie war das Auftreten einer Herzinsuffizienz und die Hospitalisationshäufigkeit für Herzinsuffizienz in der mit Losartan behandelten Gruppe signifikant geringer [33].

\section{Arteriosklerose}

Kürzlich konnte klar gezeigt werden, dass Patienten mit einem erhöhten kardiovaskulären Risiko, aber normaler linksventrikulärer Funktion auch hinsichtlich des Auftretens einer Herz- insuffizienz, signifikant von einer Behandlung mit Ramipril profitieren (HOPE-Studie) [34]. Metaanalysen deuten darauf hin, dass eine Therapie mit Plättchenaggregationshemmern die Häufigkeit einer Herzinsuffizienz vermindert [35]. Im kürzlich publizierten CURE-Trial war das Auftreten einer Herzinsuffizienz in der mit Clopidogrel behandelten Gruppe signifikant geringer als in der Placebogruppe [36].

\section{Linksventrikuläre Dysfunktion}

Die SOLVD-Studie hat klar belegt, dass bei Patienten mit asymptomatischer Einschränkung der linksventrikulären Funktion der Einsatz eines ACE-Hemmers das Auftreten einer Herzinsuffizienz abhängig vom Schweregrad der linksventrikulären Dysfunktion signifikant reduziert [15]. Deshalb sollten alle Patienten mit eingeschränkter linksventrikulärer Funktion (Auswurffraktion $<40 \%$ ) mit einem ACE-Hemmer behandelt werden, wenn keine Kontraindikation für diese Therapie besteht. Ein Follow-up über 12 Jahre konnte eine Mortalitätsreduktion von 14\% dokumentieren [37].

\section{Valvuläre Herzkrankheiten}

Das Vorliegen einer valvulären Herzkrankheit stellt ebenfalls einen prädisponierenden Faktor für die Entwicklung einer Herzinsuffizienz dar [38]. Deshalb sollten Patienten mit bekannten Vitien regelmässig kontrolliert werden. Bei Auftreten von Symptomen muss eine chirurgische Intervention erwogen werden. Bei Patienten mit Aorteninsuffizienz und Mitralinsuffizienz ist allerdings die Indikation für eine Klappenchirurgie auch bei asymptomatischen Patienten gegeben, wenn es infolge chronischer Volumenbelastung zu einer Abnahme der Auswurffraktion bzw. Vergrösserung der linken Ventrikels kommt (Aorteninsuffizienz: EF $<50 \%$ oder enddiastolischer Durchmesser des linken Ventrikels $>75 \mathrm{~mm}$; Mitralinsuffizienz: EF < 50\% oder endsystolischer Durchmesser des linken Ventrikels $>50 \mathrm{~mm}$ ). Deshalb sind regelmässige echokardiographische Kontrollen auch bei asymptomatischen Patienten mit schwerer Aorten- oder Mitralinsuffizienz indiziert [38]. Im Vergleich zu Digitalis hatte eine Behandlung mit langwirkendem Nifedipin bei Patienten mit schwerer Aorteninsuffizienz und normaler linksventrikulärer Funktion einen günstigen Einfluss auf den Verlauf [39].

Die erwähnten Beispiele zeigen, dass bei Strategien zur wirksamen Prophylaxe der im Zunehmen begriffenen Herzinsuffizienz der Früherfassung von Risikopersonen besondere Bedeutung zukommt. 


\section{Literatur}

1 Hunt SA, Baker DW, Chin MH, Cinquegrani MP, Feldmanmd AM, Francis GS, et al. ACC/AHA Guidelines for the Evaluation and Management of Chronic Heart Failure in the Adult: Executive Summary. A Report of the American College of Cardiology/American Heart Association Task Force on Practice Guidelines (Committee to Revise the 1995 Guidelines for the Evaluation and Management of Heart Failure): Developed in Collaboration With the International Society for Heart and Lung Transplantation; Endorsed by the Heart Failure Society of America. Circulation 2001;104:2996-3007.

2 Lloyd-Jones DM, Larson MG, Leip EP, Beiser A, D'Agostino RB, Kannel WB, et al. Lifetime risk for developing congestive heart failure: the Framingham heart study. Circulation 2002;106:3068-72.

3 He J, Ogden LG, Bazzano LA, Vupputuri S, Loria C, Whelton PK. Risk factors for congestive heart failure in US men and women: NHANES I epidemiologic follow-up study. Arch Intern Med 2001; 161:996-1002.

4 Levy D, Larson MG, Vasan RS, Kannel WB, Ho KK. The progression from hypertension to congestive heart failure. JAMA 1996;275:1557-62.

5 Haffner SM, Lehto S, Ronnemaa T, Pyorala K, Laakso M. Mortality from coronary heart disease in subjects with type 2 diabetes and in nondiabetic subjects with and without prior myocardial infarction. N Engl J Med 1998;339:229-34.

6 McKee PA, Castelli WP, McNamara PM, Kannel WB. The natural history of congestive heart failure: the Framingham study. N Engl J Med 1971;285:1441-6.

7 Remes J, Reunanen A, Aromaa A, Pyorala K. Incidence of heart failure in eastern Finland: a population-based surveillance study. Eur Heart J 1992; 13:588-93.

8 Kannel WB, Hjortland M, Castelli WP. Role of diabetes in congestive heart failure: the Framingham study. Am J Cardiol 1974;34:29-34.

9 Shindler DM, Kostis JB, Yusuf S, Quinones MA, Pitt B, Stewart D, et al. Diabetes mellitus, a predictor of morbidity and mortality in the Studies of Left Ventricular Dysfunction (SOLVD) Trials and Registry. Am J Cardiol 1996;77:1017-20.

10 Kenchaiah S, Evans JC, Levy D, Wilson PW, Benjamin EJ, Larson MG, et al. Obesity and the risk of heart failure. N Engl J Med 2002;347:305-13.

11 Gerstein HC, Mann JF, Yi Q, Zinman B, Dinneen SF, Hoogwerf B, et al. Albuminuria and risk of cardiovascular events, death, and heart failure in diabetic and nondiabetic individuals. JAMA 2001;286:421-6.

12 McKelvie RS, Benedict CR, Yusuf S. Evidencebased cardiology: prevention of congestive heart failure and management of asymptomatic left ventricular dysfunction. Br Med J 1999; 318:1400-2.

13 Benedict CR, Shelton B, Johnstone DE, Francis G Greenberg B, Konstam M, et al. Prognostic significance of plasma norepinephrine in patients with asymptomatic left ventricular dysfunction. SOLVD Investigators. Circulation 1996;94:690-7.
14 Remme WJ, Swedberg K. Guidelines for the diagnosis and treatment of chronic heart failure. Eur Heart J 2001;22:1527-60.

15 Wang TJ, Levy D, Benjamin EJ, Vasan RS. The epidemiology of «asymptomatic» left ventricular systolic dysfunction: implications for screening. Ann Intern Med 2003;138:907-16.

16 Effect of enalapril on survival in patients with reduced left ventricular ejection fractions and congestive heart failure. The SOLVD Investigators. N Engl J Med 1991;325:293-302.

17 Pfeffer MA, Lamas GA, Vaughan DE, Parisi AF, Braunwald E. Effect of captopril on progressive ventricular dilatation after anterior myocardial infarction. N Engl J Med 1988;319:80-6.

18 Sharpe N, Murphy J, Smith H, Hannan S. Preventive treatment of asymptomatic left ventricular dysfunction following myocardial infarction. Eur Heart J 1990;11(Suppl B):147-56.

19 Kober L, Torp-Pedersen C, Carlsen JE, Bagger H, Eliasen P, Lyngborg K, et al. A clinical trial of the angiotensin-converting-enzyme inhibitor trandolapril in patients with left ventricular dysfunction after myocardial infarction. Trandolapril Cardiac Evaluation (TRACE) Study Group. N Engl J Med 1995;333:1670-6.

20 Kober L, Bloch Thomsen PE, Moller M, TorpPedersen C, Carlsen J, Sandoe E, et al. Effect of dofetilide in patients with recent myocardial infarction and left-ventricular dysfunction: a randomised trial. Lancet 2000;356:2052-8.

21 Dahlof B, Lindholm LH, Hansson L, Schersten B, Ekbom T, Wester PO. Morbidity and mortality in the Swedish Trial in Old Patients with Hypertension (STOP-Hypertension). Lancet 1991;338: 1281-5.

22 Hansson L, Lindholm LH, Ekbom T, Dahlof B, Lanke J, Schersten B, et al. Randomised trial of old and new antihypertensive drugs in elderly patients: cardiovascular mortality and morbidity the Swedish Trial in Old Patients with Hypertension-2 study. Lancet 1999;354:1751-6.

23 Kostis JB, Davis BR, Cutler J, Grimm RH Jr., Berge KG, Cohen JD, et al. Prevention of heart failure by antihypertensive drug treatment in older persons with isolated systolic hypertension. SHEP Cooperative Research Group. JAMA 1997;278:212-6.

24 Staessen JA, Fagard R, Thijs L, Celis H, Arabidze GG, Birkenhager WH, et al. Randomised doubleblind comparison of placebo and active treatment for older patients with isolated systolic hypertension. The Systolic Hypertension in Europe (SystEur) Trial Investigators. Lancet 1997;350:757-64.

25 Brown MJ, Palmer CR, Castaigne A, de Leeuw PW, Mancia G, Rosenthal T, et al. Morbidity and mortality in patients randomised to double-blind treatment with a long-acting calcium-channel blocker or diuretic in the International Nifedipine GITS study: Intervention as a Goal in Hypertension Treatment (INSIGHT). Lancet 2000; 356:366-72.

26 Major cardiovascular events in hypertensive patients randomized to doxazosin vs chlorthalidone: the antihypertensive and lipid-lowering treatment to prevent heart attack trial (ALLHAT). ALLHAT Collaborative Research Group. JAMA 2000; 283:1967-75. 
27 Major outcomes in high-risk hypertensive patients randomized to angiotensin-converting enzyme inhibitor or calcium channel blocker vs diuretic: The Antihypertensive and Lipid-Lowering Treatment to Prevent Heart Attack Trial (ALLHAT). JAMA 2002;288:2981-97.

28 Dahlof B, Devereux RB, Kjeldsen SE, Julius S, Beevers G, Faire U, et al. Cardiovascular morbidity and mortality in the Losartan Intervention For Endpoint reduction in hypertension study (LIFE): a randomised trial against atenolol. Lancet 2002; 359:995-1003.

29 Lüscher TF, Noll G. Hypertonie-Management 2003: Confudes at a higher level? Kardiovask Med 2003;6:98-103.

30 Pahor M, Psaty BM, Alderman MH, Applegate WB, Williamson JD, Cavazzini C, et al. Health outcomes associated with calcium antagonists compared with other first-line antihypertensive therapies: a meta-analysis of randomised controlled trials. Lancet 2000;356:1949-54.

31 Kjekshus J, Pedersen TR, Olsson AG, Faergeman $\mathrm{O}$, Pyorala K. The effects of simvastatin on the incidence of heart failure in patients with coronary heart disease. J Card Fail 1997;3:249-54.

32 Tight blood pressure control and risk of macrovascular and microvascular complications in type 2 diabetes: UKPDS 38. UK Prospective Diabetes Study Group. Br Med J 1998;317:703-13.

33 Brenner BM, Cooper ME, de Zeeuw D, Keane WF, Mitch WE, Parving HH, et al. Effects of losartan on renal and cardiovascular outcomes in patients with type 2 diabetes and nephropathy. N Engl J Med 2001;345:861-9.

34 Yusuf S, Sleight P, Pogue J, Bosch J, Davies R, Dagenais G. Effects of an angiotensin-convertingenzyme inhibitor, ramipril, on cardiovascular events in high-risk patients. The Heart Outcomes Prevention Evaluation Study Investigators. N Engl J Med 2000;342:145-53.

35 Collaborative meta-analysis of randomised trials of antiplatelet therapy for prevention of death, myocardial infarction, and stroke in high risk patients. Br Med J 2002;324:71-86.

36 Yusuf S, Zhao F, Mehta SR, Chrolavicius S, Tognoni G, Fox KK. Effects of clopidogrel in addition to aspirin in patients with acute coronary syndromes without ST-segment elevation. N Engl J Med 2001;345:494-502.
37 Jong P, Yusuf S, Rousseau MF, Ahn SA, Bangdiwala SI, Effect of enalapril on 12-year survival and life expectancy in patients with left ventricular systolic dysfunction: a follow-up study. Lancet 2003; 361:1843-8

38 Bonow RO, Carabello B, de Leon AC Jr., Edmunds LH Jr., Fedderly BJ, Freed MD, et al. Guidelines for the management of patients with valvular heart disease: executive summary. A report of the American College of Cardiology/American Heart Association Task Force on Practice Guidelines (Committee on Management of Patients with Valvular Heart Disease). Circulation 1998;98:1949-84.

39 Scognamiglio R, Rahimtoola SH, Fasoli G, Nistri S, Dalla Volta S. Nifedipine in asymptomatic patients with severe aortic regurgitation and normal left ventricular function. N Engl J Med 1994; 331:689-94.

40 Hansson L, Hedner T, Lund-Johansen P, Kjeldsen SE, Lindholm LH, Syvertsen JO, et al. Randomised trial of effects of calcium antagonists compared with diuretics and beta-blockers on cardiovascular morbidity and mortality in hypertension: the Nordic Diltiazem (NORDIL) study. Lancet 2000; 356:359-65

41 Hansson L, Lindholm LH, Niskanen L, Lanke J, Hedner T, Niklason A, et al. Effect of angiotensinconverting-enzyme inhibition compared with conventional therapy on cardiovascular morbidity and mortality in hypertension: the Captopril Prevention Project (CAPPP) randomised trial. Lancet 1999;353:611-6.

42 Wing LM, Reid CM, Ryan P, Beilin LJ, Brown MA, Jennings GL, et al. A comparison of outcomes with angiotensin-converting-enzyme inhibitors and diuretics for hypertension in the elderly. N Engl J Med 2003;348:583-92.

43 Lindholm LH, Ibsen H, Dahlof B, Devereux RB, Beevers G, de Faire U, et al. Cardiovascular morbidity and mortality in patients with diabetes in the Losartan Intervention For Endpoint reduction in hypertension study (LIFE): a randomised trial against atenolol. Lancet 2002;359:1004-10.

\section{Gstaader Treffen 2004 \\ der Schweizerischen Herzstiftung \\ 29. Januar bis 1. Februar 2004}

Weitere Informationen finden Sie unter: www.congress-info.ch/gstaad2004/

Patronat Kongress: Schweizerische Herzstiftung und Swiss Society of Cardiology

Hauptsponsor Kongress: AstraZeneca

Co-Sponsor Kongress: EMH Schweizerischer Ärzteverlag AG 